\title{
Zur Schlichtung des Streites zwischen Haldane und Mosso bezüglich der Höhen-Hypokapnie
}

Zugleich ein Beitrag zur besseren Gasdiffusion im Unterdruck*

Von Karl Lenggenhager

Mossos Theorie: In der Höhe und im Unterdruck verliert der Mensch auf physikalischem Wege vermehrt Kohlensäure als unmittelbare Folge der Luftdruckabnahme. Dies geschehe ungefähr so wie bei einer $\mathrm{CO}_{2}$-haltigen Mineralwasserflasche, welche beim Öffnen ihres Schlusses plötzlich $\mathrm{CO}_{2}$ Blasen austreten läßt.

Haldanes Ansicht: Die in der Höhe oder im Unterdruck feststellbare Verringerung der alv. $\mathrm{CO}_{2}$ ist eine rein sekundäre Erscheinung, bedingt durch das infolge $\mathrm{O}_{2}$-Mangels leicht vergrößerte Atemvolumen.

Haldane berief sich auf die bekannten Gesetze, welche besagen, daß die Dampfspannung einer verdunstungsfähigen Lösung bei gegebener Temperatur unabhängig von der allfälligen Anwesenheit anderer Gase ist. Er fühlte sich in seiner Ansicht noch bestärkt, weil er in Eigenversuchen in der Unterdruckkammer bei $300 \mathrm{~mm} \mathrm{Hg}$ fast keine Verminderung der alveolären $\mathrm{CO}_{2}$-Spannung mehr feststellen konnte, wenn reiner $\mathrm{O}_{2}$ geatmet wurde (s. auf S.222 seines Buches «Respiration» und in der Originalarbeit von Boycott u. Haldane v. 1908). (Atemvolumina, die sich nach eigenen Untersuchungen sogar unter die Norm stellen, wurden nicht gemessen!)

Vergeblich suchte Haldane in persönlicher Unterredung Mosso von dessen «Irrtum» abzubringen, und so starb Mosso, bis zuletzt von seiner Ansicht der primären «Akapnie» überzeugt.

Haldane hat dann auch in seinem Buche «Respiration» auf S. 302 in sonst ungewohnter Schärfe Mossos Haltung mit den Worten kritisiert: "His physical chemistry was completely at fault."

Anderseits gibt Haldane in seinem Buche «Respiration» 1935 auf S. 224 an (Mosso war schon lange gestorben), daß in verdünnter Luft von $368 \mathrm{~mm}$

\footnotetext{
* Diese Arbeit ist ein kleiner Teilausschnitt unserer während 16 Jahren im Forschungsinstitut Jungfraujoch $(3450 \mathrm{~m})$ jeweils während 14 Tagen in den Frühjahrsferien mit 8-14 gesunden Versuchspersonen durchgeführten und im Tale ergänzten Untersuchungen (damaliger Leiter: Prof. Dr. A.v. Muralt, Bern).
} 
$\mathrm{Hg}$ wegen offenbar rascherer Diffusion des $\mathrm{O}_{2}$ in der Lungenluft geringere Störungen als erwartet auftreten (!), ohne aber auf rascheres Abrauchen der Blut- $\mathrm{CO}_{2}$ in die Lunge zu schließen.

Versuche zur Demonstration eines im Unterdruck rascher erfolgenden Gasausgleichs in der Lungenluft

a) In unserer Unterdruckkammer führte unser Doktorand K.Zürcher folgende Versuche aus:

21 gesunde Versuchspersonen mußten nüchtern je eine Stunde lang in der geöffneten Kammer, die gut durchlüftet wurde, möglichst bewegungsarm vorliegen. 1/4 Stunde vor Versuchsbeginn wurde eine Nasenklemme zur Gewöhnung an die Mundatmung angelegt. Hernach wurde ihr Ruheatemvolumen durch immer die gleiche Gasuhr bestimmt. Dies wurde solange eingeübt, bis ein nahezu regelmäßiges Minuten-Atemvolumen erreicht wurde, welches dann während 10 Minuten weiterhin gemessen wurde. Hernach wurde ein Unterdruck, entsprechend der Höhe von Jungfraujoch $(3450 \mathrm{~m}=490 \mathrm{~mm} \mathrm{Hg})$ hergestellt und der Atmungsversuch während gleich langer Zeit aus in die Kammer verbrachten Luft-gefüllten Gummisäcken wiederholt.

So ergab sich im Durchschnitt ein von 5,65 l/min geatmetes Atemvolumen im Normaldruck, das im Unterdruck auf $6,23 \mathrm{l} / \mathrm{min}$ gesteigert wurde. Dies entspricht einer Zunahme von nur 0,58 1/min, was einer Steigerung von nur $11,02 \%$ entspricht, wiewohl der $\mathrm{O}_{2}$-Druck im Verhältnis von $710 \mathrm{~mm}$ $\mathrm{Hg}$ (Tal) auf $490 \mathrm{~mm}(« \mathrm{Joch} »)$ gesunken war, also von 100 auf $69 \%$, siehe Tabelle 1. Man würde eigentlich eine um $31 \%$ erhöhte Atmung erwarten, wenn pro Zeiteinheit gleich viel Molekel ins Blut aufgenommen würden. In Wirklichkeit beträgt die Erhöhung des Atemvolumens aber nur 11,02\%.

Tab.1. In der Unterdruckkammer entsprechend Jungfraujochhöhe $(3450 \mathrm{~m})$ werden die Ruhe-Atemvolumina nur um 11,02\% vermehrt, verglichen zur Talatmung $(560 \mathrm{~m})$, wiewohl der $\mathrm{O}_{2}$ sich von 100 auf $69 \%$ verringert hat.

\begin{tabular}{|l|l|l|l|}
\hline $\begin{array}{l}\text { Minuten-Atemvolumen } \\
\text { nach 10-12' Atmung }\end{array}$ & $\begin{array}{l}\text { Bern, 710 mm Hg } \\
\text { Minuten-Atem- } \\
\text { Volumina }\end{array}$ & $\begin{array}{l}\text { Unterdruckkammer } \\
\text { im Tal. } 490 \mathrm{~mm} \mathrm{Hg} \\
(=3450 \mathrm{~m})\end{array}$ & $\begin{array}{l}\text { Prozentuale } \\
\text { Steigerung }\end{array}$ \\
\cline { 2 - 4 } $\begin{array}{l}\text { Mittelwerte von } \\
21 \text { Medizinstudenten }\end{array}$ & $5,65 \mathrm{l} / \mathrm{min}$ & $6,23 \mathrm{l} / \mathrm{min}$ & $11,02 \%$ \\
\hline
\end{tabular}


Tab.2. Zeigt die um 22,31 1/min gesteigerte Ruheventilation, wenn im Tal $\mathrm{O}_{2}$-arme Luft entsprechend der Höhe von Jungfraujoch $(3450 \mathrm{~m})$ geatmet wurde. Durchschnitt aus 27 Einzelversuchen mit 19 gesunden Versuchspersonen. (Auch hier blieben die Atemfrequenzen praktisch stabil.)

\begin{tabular}{|l|l|l|l|}
\hline $\begin{array}{l}\text { Durchschnittswerte } \\
\text { von } 27 \text { Versuchen an } \\
19 \text { gesunden Versuchs- } \\
\text { personen }\end{array}$ & $\begin{array}{l}\text { Talluft } \\
\text { Atemvol. in } / \mathrm{min}\end{array}$ & $\begin{array}{l}\text { «Jochluft» im Tal } \\
\text { Atemvol. in 1/min }\end{array}$ & $\begin{array}{l}\text { Prozentuale } \\
\text { Steigerung }\end{array}$ \\
\cline { 2 - 4 } & $5,02 \mathrm{l}$ & $6,20 \mathrm{l}$ & $22,31 \%$ \\
\hline
\end{tabular}

Wenn man diese Werte auf Meereshöhe und $0^{\circ} \mathrm{C}$ reduziert, so sind die Minuten-Volumina im Unterdruck sogar erheblich kleiner als im Normaldruck, nämlich von 4,85 l/min bei Normaldruck auf 3,56 lim Unterdruck von $490 \mathrm{~mm} \mathrm{Hg}$ gesunken!

Daraus ergibt sich also eine ökonomischere Verwertung der Atemluft im Unterdruck, was zu Gunsten Mossos spricht.

b) Atmeten wir umgekehrt im Tal ein Luftgemisch, das den gleichen $\mathrm{O}_{2^{-}}$ Druck aufwies wie auf Jungfraujoch, so ergab sich aus 27 Versuchen mit 19 Studenten (Dissertation J.Pfenninger) eine durchschnittliche Steigerung des Tal-Ruheatemvolumens von 5,02 $\mathrm{l} / \mathrm{min}$ auf $6,20 \mathrm{l} / \mathrm{min}$, entsprechend einer um 22,31 \%igen Steigerung, siehe Tab.2.

Schon Haldane beschrieb solche Versuche, die gleichsinnig verliefen (S. 183), ohne dies jedoch in Verbindung zu Mossos Ansicht zu bringen.

Diese Versuche zeigen die zeitliche Erschwerung der $\mathrm{O}_{2^{-}}$und $\mathrm{CO}_{2^{-}}$ Diffusion durch die dichtere $\mathrm{N}_{2}$-Molekülschicht des Tales in der Lunge und stützen wieder (diesmal im umgekehrten Sinn) die Vorstellung einer Abhängigkeit der Gasdiffusionsgeschwindigkeit von der Dichte der Gasschichten: Die zahlreichen $\mathrm{N}_{2}$-Molekel erschweren den Zutritt der verminderten $\mathrm{O}_{2}$ Molekel in der Lungenluft. Deshalb muß das Atem-Minutenvolumen gesteigert werden.

Schon Paul Bert hatte 1878 gefunden, daß Vögel, welche im Unterdruck die ersten Symptome von $\mathrm{O}_{2}$-Mangel aufweisen, augenblicklich starben, wenn der Druck durch reinen $\mathrm{N}_{2}$ sofort normalisiert wurde (ref. bei Haldane, «Respiration», S. 301!).

c) Für Mossos Auffassung eines rascheren Gasaustausches im Unterdruck sprechen nun auch folgende Eigenversuche an 19 eingeübten Mithelfern. Deren Atemvolumina verkleinerten sich von $5,02 \mathrm{l} / \mathrm{min}$ auf 4,48 l, wenn im Unterdruck von $490 \mathrm{~mm} \mathrm{Hg}$ (Jochhöhe) die $\mathrm{O}_{2}$-Spannung gleich gehalten wurde wie im Normaldruck, siehe Tab.3. 
Tab.3. Zeigt das um 10,7\% verkleinerte Ruheatemvolumen in der Unterdruckkammer entsprechend Jochhöhe $(3450 \mathrm{~m})$ bei Atmung gleicher $\mathrm{O}_{2}$-Spannung wie im Tal.

\begin{tabular}{|l|l|l|}
\hline $\begin{array}{l}\text { Atemvolumen, unreduziert } \\
\text { min/l Durchschnitt }\end{array}$ & Talluft & Tal- $\mathrm{O}_{2}$-Druck im Unter- \\
aus 19 Versuchspersonen & $5,02 \mathrm{l}$ & druck $(3450 \mathrm{~m})=4,48 \mathrm{l} / \mathrm{min}$ \\
& pro Minute & $(=-10,75 \%$ weniger als in Talluft $)$ \\
\hline
\end{tabular}

Die in der Lit. recht unterschiedlichen Angaben über zu große und zu kleine Atemvolumina in der Höhe (siehe z.B. bei F.v.Tavel, oder bei H.Pauli) beruhen sicher auf ungenügend ausgeruhten oder ungenügend eingeübten Versuchspersonen, so daß z.B. Atemvolumina im Tal von 10-14 1/min angegeben werden. Der Physiologe Luft gibt die Atemsteigerung auf Joch $(3450 \mathrm{~m})$ «trotz großen Schwankungen» um 25-30\% an. Dagegen beruhen auch die leichter erhöhten Berg-Atemvolumina von Loewy auf der Tatsache, daß seine Untersuchungen erst am zweiten Höhentage ausgeführt werden konnten, wo die Atemvolumina sich deutlich vergrößern.

In ganz ähnlichem Sinne sprechen unsere Versuche auf Jungfraujoch (3450 m) an 13 gesunden Versuchspersonen (Studenten und Assistenten). Die Durchschnittswerte (10-12minütiger Ruheatemvolumina) pro Minute wurden 1-5 Stunden nach Ankunft auf Jochhöhe unter Atmung normaler, den Talwerten entsprechenden $\mathrm{O}_{2}$-Spannungen bestimmt und mit den TalRuheatemvolumina des Vortages (infolge der frühmorgendlichen Abreise) verglichen. Dies ergab eine Senkung auf 4,36 l/min auf Joch gegenüber den Talwerten von $4,74 \mathrm{l} / \mathrm{min}$, also um 10,96\% gesenktes Atemvolumen gegenüber dem Tal, siehe Tab. 4 .

Es ist wichtig, daß solche Versuche gleich am Ankunftstage auf Joch ausgeführt werden, da schon über Nacht durch Verminderung der im Urin ausgeschiedenen Reservealkalien und infolge Einstrom von Milchsäure und 2,3-Phosphoglyzerin (nach Finch und Lenfant) ins Blut das Atemvolumen eindeutig vergrößert wird bei Verminderung des AtemanhalteVermögens um ca. 1/4 (siehe Beitrag im Universum).

Tab.4. Auch bei künstlich gleich gehaltener $\mathrm{O}_{2}$-Spannung auf Jochhöhe wie im Tal ist das Ruhe-Atemvolumen am ersten Höhentage bedeutend kleiner als im Tal dank rascherem Gasaustausch im Unterdruck. Verminderung um 11\%. Dagegen gleich starke Steigerung gegenüber der Talatmung bei Atmung von Jochluft.

\begin{tabular}{|l|l|l|l|}
\hline $\begin{array}{l}\text { Unreduz. Atemvolumen } \\
\text { in min/l }\end{array}$ & $\begin{array}{l}\text { Talluft } \\
=4,74 \mathrm{l} / \mathrm{min}\end{array}$ & \multicolumn{2}{|c|}{$\begin{array}{c}\text { Jungfraujoch } \\
\text { Ankunftstag }\end{array}$} \\
& & $\begin{array}{l}\text { «Tal-O } \\
=4,36 \mathrm{l} / \mathrm{min}\end{array}$ & $\begin{array}{l}\text { Jochluft } \\
=5,23 \mathrm{l} \\
(=-11 \%)\end{array}$ \\
& & $(=+11 \%)$ \\
\hline
\end{tabular}


d) Schließlich darf die zeitliche Beanspruchung der Gasdiffusion in diesem Zusammenhang auch für folgende, bekannte und durch Eigenversuche voll bestätigte Tatsache angeführt werden: Am Ende eines Atemanhalteversuches bläst man die Luft in einen leeren Gummisack und reaspiriert diese Luft nochmals. Stets bei gebieterischer Atemnot wird die Luft wieder in den leeren Sack ausgeatmet und gleich wieder inspiriert. Diese AtemanhalteZeiten werden so immer kürzer. Addiert aber ergeben sich so doppelt so lange Atem-Anhaltemöglichkeiten verglichen mit den einfachen AtemanhalteVersuchen. Dies geschieht auch bei fortlaufenden Pendelatmungen nach dem ersten Atem-Anhalteversuch, siehe Tab. 5.

Tab. 5 zeigt, daß die nach maximalem Atemanhalteversuch in leeren Gummisack exspirierte Luft noch ungefähr gleich lange ein- und ausgeatmet werden kann (Pendelatmung), bis wieder gebieterische Atemnot auftritt. Erst jetzt darf mit «maximaler» Ausnützung der Atemluft gerechnet werden (Dissertation meines Schülers Y. Rosenstrauch).

\begin{tabular}{|l|l|l|}
\cline { 2 - 3 } \multicolumn{1}{c|}{} & $\begin{array}{l}\text { Max. Atemanhaltezeit } \\
\text { in Sekunden }\end{array}$ & $\begin{array}{l}\text { Anschließende } \\
\text { Pendelatmungszeit } \\
\text { in Sekunden }\end{array}$ \\
\hline $\begin{array}{l}\text { Durchschnittswerte von } \\
21 \text { Versuchspersonen }\end{array}$ & $\begin{array}{l}73 \\
\text { Sekunden }\end{array}$ & $\begin{array}{l}72 \\
\text { Sekunden }\end{array}$ \\
\hline
\end{tabular}

Diese Versuche zeigen sehr eindrücklich, daß es für die Diffusion der Gase in der Lungenluft einer bestimmten Zeit bedarf, die so groß ist, daß es in der ruhig gestellten Lunge im Moment des gebieterischen Atemimpulses nach dem Anhalteversuch noch lange nicht zur idealen Durchmischung der Lungenluft mit der Totraumluft durch Diffusion gekommen ist! Es stehen eben die größeren und trägeren $\mathrm{CO}_{2}-\mathrm{Molekel}$ als dichtere Barriere dem freien Gasaustausch im Wege und bedingen einen $\mathrm{CO}_{2}$-Rückstau im Blute.

Haldanes Erklärung (1935) für diese Beobachtungen ist eine andere: Er nimmt an, daß normalerweise nie alle Lungenteile gleichmäßig durchlüftet seien, wodurch sogenannte innere Kurzschlüsse zwischen arteriellem und venösem Lungenblut resultieren. Jedoch scheint diese für Ruhepausen berechtigte Meinung für in maximalen Inspirationsstellungen gehaltenen Lungen kaum zutreffend.

e) Die erhebliche Trägheit der Gasdiffusion bei Normaldruck geht übrigens aus der bekannten Erscheinung des Hechelns der Hunde hervor, die auf diese Weise zwar die Temperaturregulierung ihres Körpers bedingen, jedoch durchaus nicht hyperventilationstetanisch werden. 
In einer Inaugurations-Dissertation unseres Schülers R.Schmid haben wir dieses Hecheln durch gesunde Versuchspersonen während je 1-3 Minuten imitiert. Trotz Steigerung der Atemvolumina um das $3 \frac{1}{2}-4 \frac{1}{2}$ fache konnte es sogar zu einem leichten $\mathrm{CO}_{2}$-Anstieg der alveolaren $\mathrm{CO}_{2}$ kommen (um $1 \%)$.

f) Bei Atmung im Tal stieg das in reinem $\mathrm{O}_{2}$ geatmete Ruhevolumen nach Untersuchungen meines Dissertanten J.Pfenninger an 20 normalen Versuchspersonen von $24-25 \mathrm{Jahren}$ von $5,35 \mathrm{l} / \mathrm{min}$ auf $5,91 \mathrm{l}$, und die alv. $\mathrm{CO}_{2}$ sank von 40 auf $38 \mathrm{~mm} \mathrm{Hg}$.

Schon Haldane fand diese Zunahme. Er erklärte sie durch die leicht saure Wirkung des $\mathrm{Oxy}-\mathrm{Hb}$, das die Aufnahme der Gewebs- $\mathrm{CO}_{2}$ erschwert.

Dagegen sank das Atemvolumen dieser Versuchspersonen bei halbem Barometerdruck in unserer Unterdruckkammer bei reiner $\mathrm{O}_{2}$-Atmung von 5,35 l/min auf 5,07 l/min ab, und die alv. $\mathrm{CO}_{2}$ sank auf $37 \mathrm{~mm} \mathrm{Hg}$ (gegen $40 \mathrm{~mm}$ bei normaler Luftatmung). Dies steht im Gegensatz zum vergrößerten Atemvolumen bei reiner $\mathrm{O}_{2}$-Atmung im Tal.

Zur Erklärung muß angenommen werden, daß es in der verdünnten Lungenluft zu einer rascheren Diffusion des $\mathrm{O}_{2}$ und der $\mathrm{CO}_{2}$ kommt. (Hier spielt der im Tal bei reiner $\mathrm{O}_{2}$-Atmung beobachtete Rückstau der Gewebs$\mathrm{CO}_{2}$ durch das leicht saure $\mathrm{Oxy}-\mathrm{Hb}$ nur noch eine geringe Rolle, so daß es doch zu einer deutlichen Verkleinerung des Atemvolumens kommt.)

Haldane beschrieb in anderem Zusammenhang in seinem Buche «Respiration», daß eine Kerzenflamme in einem gegebenen Luftvolumen im Tal nach dem Erlöschen noch einen $\mathrm{O}_{2}$-Gehalt von ca. $17 \%$ in dem Raum hinterläßt. Trotzdem brennt diese Kerze jedoch wieder eine geraume Zeit, wenn sie mit Frischluft im halben Unterdruck angezündet wird, der nur noch $10 \% \mathrm{O}_{2}$ zur Verfügung hat, yerglichen mit den $17 \% \mathrm{O}_{2}$ der Tal-Verbrennungsluft (was wir voll bestätigen konnten).

Haldanes Erklärung ist folgende: Im Unterschied zum Menschen richtet sich die Flamme nicht nach dem Partialdruck des $\mathrm{O}_{2}$, sondern nur nach dessen Prozentsatz.

Gegen diese Vorstellung sprechen z. T. folgende Untersuchungen meines Doktoranden A. Schütz (und eigene): Die Brenndauer einer Kerzenflamme unter immer derselben Glasglocke brennt im Tal 23" lang; auf Jungfraujoch 17,5 "; in «Jochluft» im Tal nur 5"! in «Talluft» auf Joch 34"!

Wird aber aller Luftstickstoff der Talluft durch $\mathrm{CO}_{2}$ ersetzt, so erlischt die Flamme trotz normalem $\mathrm{O}_{2}$-Gehalt innert einer Sekunde! 
g) Am Schluß der physiologischen Versuche sei noch kurz folgender Eigenversuch angeführt: Während in liegender Lage mein Atem unter Normaldruck 122 Sek. lang angehalten werden konnte (Mittelwert aus 3 Versuchen), sank dieser Wert (zunächst unerwarteterweise) auf 95 Sek., wenn die gleichen Versuche im sofort anschließenden Unterdruck von $1 / 2$ Atm bei gleich gehaltener $\mathrm{O}_{2}$-Spannung wie im Normaldruck ausgeführt wurden. Dabei ergaben sich gegen Ende der Versuche früher auftretende Einziehungen des Zwerchfells gegen den Thoraxraum.

Die Erklärung hierfür wird sein, daß die absolute Verkleinerung des gleichen Atemraumes im Unterdruck gegen Ende der Versuche doppelt so stark ist als bei Normaldruck; denn es wird weiterhin $\mathrm{O}_{2}$ in normaler Menge, jedoch im Doppel seines Volumens verschwinden, während keine $\mathrm{CO}_{2}$ mehr in die Lungenluft diffundieren kann, wenn deren Konzentration 20-22\% (bei $1 / 2$ Atm Druck) erreicht hat. So muß es gegen Ende des Versuches bei weiterer $\mathrm{O}_{2}$-Abnahme zu einer rascheren $\mathrm{CO}_{2}$-Konzentration kommen durch Verkleinerung des Lungenraumes mit Rückstrom des sich konzentrierenden $\mathrm{Co}_{2}$ ins Blut.

Ein Analogon hierzu bietet ein früher ausgeführter Eigenversuch, wobei am Ende einer gewöhnlichen Expiration die Lungen mit leicht resorbierbarem Acetylengas maximal aufgefüllt wurden. Dieser Atemanhalteversuch mußte jedoch schon nach 20 " unterbrochen werden, da sich eine ungewöhnliche und stärkste Atemnot einstellte, die eine länger anhaltende, allergrößte Atemnot mit größter Atmung bedingte und mich beinahe ersticken ließ, bis alle $\mathrm{CO}_{2}$ wieder ausgeatmet war. Infolge sehr rascher Resorption des Acetylens häufte sich die $\mathrm{CO}_{2}$ infolge rascher Verkleinerung des Lungenraumes in gefährlicher Weise an, wie wenn z. B. ein $40 \%$ iges $\mathrm{CO}_{2}$-Luftgemisch geatmet worden wäre.

\section{Zusammenfassung}

Der alte Streit zwischen Haldane und Mosso betreffend die Frage eines primär im Unterdruck verstärkten «Abrauchens» der Blut- $\mathrm{CO}_{2}$ (Ansicht Mossos) im Gegensatz zum sekundären $\mathrm{CO}_{2}$ Verlust infolge der durch $\mathrm{O}_{2}$-Mangel bedingten Hyperpnoe (Haldanes Ansicht) wurde experimentell in der Unterdruckkammer und in zahlreichen Expeditionen auf Jungfraujoch $(3450 \mathrm{~m})$ mit vielen, eingeübten Versuchspersonen geprüft.

Dabei hat sich herausgestellt, daß doch die Mosso'sche Ansicht mitverantwortlich ist für einen Teil des in der Höhe oder im Unterdruck erfolgenden $\mathrm{CO}_{2}$-Verlustes, der nicht nur durch vergrößerte Atmung bedingt ist, sondern auch durch erleichterte Diffusion im Unterdruck.

So ergaben zahlreiche Untersuchungen in der Unterdruckkammer bei $490 \mathrm{~mm} \mathrm{Hg}$ 
(entsprechend dem auf Jungfraujoch bei unseren dortigen Untersuchungen festgestellten Druck), daß bei Innehaltung normaler $\mathrm{O}_{2}$-Spannung der Atemluft wie im Tal das Ruheatemvolumen eingeübter, ausgeruhter Versuchspersonen um 11,02\% verkleinert wird gegenüber dem Ruheatemvolumen im Tal.

Deshalb ist auch das Ruhe-Atemvolumen nüchterner, ausgeruhter Versuchspersonen nach zahlreichen Eigenversuchen am ersten Höhentage auf Jungfraujoch nur um 11,0\% vergrößert, trotzdem die $\mathrm{O}_{2}$-Spannung um $30 \%$ erniedrigt ist.

Umgekehrt aber vergrößert sich das Tal-Atemvolumen um $12,35 \%$, wenn die $\mathrm{O}_{2}$ Spannung gleich niedrig gehalten wird wie auf Jungfraujoch.

Aus dem «Gleichbleiben» der alveolären $\mathrm{CO}_{2}$-Spannung bei $\mathrm{O}_{2}$-Atmung im Unterdruck durfte Haldane Mossos Ansicht eines besseren Abdiffundierens der $\mathrm{CO}_{2}$ nicht bestreiten, da er nicht gleichzeitig mit der Bestimmung der alv. $\mathrm{CO}_{2}$ das deutlich verkleinerte Atemvolumen gegenüber der Norm gemessen hat. Auch die viel längere Brenndauer einer Kerzenflamme in halbem Unterdruck, aber normaler $\mathrm{O}_{2}$-Spannung, sprechen für raschere Gasdiffusion im Unterdruck.

Damit dürfte für Mossos Ansicht eine gewisse Rehabilitierung stattgefunden haben.

\section{Literatur}

Bert P.: La Pression Barométrique, S. 749, Paris 1878; zit. auch bei Haldane J. S.: Respiration, S.301.

Boykott A.E. und Haldane J.S.: J.Physiol 37, 355; 1908.

Finch C.A. und Lenfant C.: Oxygen transport in man. New England Journ. Med. 286, 407; 1972.

Haldane J.S. und Priestley J. G.: Respiration, Oxford 1935.

Lenggenhager K.: Die Höhenkrankheit. Universum 34, 9; 1979.

Loewy A.: Physiol. des Höhenklimas. S. 169, Springer Berlin 1932.

Luft U. C.: Erg. Physiol. 44, 256; 1941.

Mosso A.: Life of men in the high alps. London, translated from second Italian Edition 1898. Opitz E.: Erg. Physiol. 44, 325; 1 p. 41.

Pauli H.G.: Pflügers Archiv 278, 447; 1964.

Pfenninger J.: Das Verhalten der alv. $\mathrm{CO}_{2}$ im Unterdruck bei Luft- und $\mathrm{O}_{2}$-Atmung beim Menschen. Inaug. Diss. Bern, 1968.

Rosenstrauch Y.: Dauer der Pendelatmung nach max. Atemretention und Verlängerung der Atemretention nach Lauf an Ort. Inaug. Diss. Bern, 1968.

Schmid R.: Beitrag zur Diffusionsgeschwindigkeit von $\mathrm{O}_{2}$ und $\mathrm{CO}_{2}$ in der Lungenluft. Inaug. Diss. Bern, 1972.

Schütz A.: Die Gültigkeit des chemischen Massenwirkungsgesetzes im Gaswechsel des Menschen. Inaug. Diss. Bern, 1942.

v. Tavel F.: Verhandl. d. freien Vereinig. Schweiz. Physiologen, Juni 1936. Die Auswirkungen des Sauerstoffmangels auf den menschl. Organism. b. kurzfristigem Aufenthalt in großer Höhe. Benno Schwabe-Verlg. 1943. Helv. Physiol. Act. Suppl. I, 1943.

Zürcher A.: Über die Differenz zwischen physikalisch berechtigter und tatsächlicher Atemsteigerung in der Höhe. Inaug. Diss. Bern, 1968. 


\section{Summary}

The old controversy between Mosso and Haldane concerning a faster diffusion of the carbonic acid at high altitudes (Mosso), and the greater loss of carbonic acid due to a greater ventilationrate at high altitudes or in lowpressure-chambre (view of Haldane) was tested in our lowpressure-chamber and on Jungfraujoch $(3450 \mathrm{~m})$ with many well trained students and assistents.

When in an underpressure-chambre or at the first day on Jungfraujoch, the pressure of oxygen was kept normal as in the valley-laboratory $(540 \mathrm{~m})$, the ventilation rate sank on the average by $11 \%$, compared with normal air and pressure at $540 \mathrm{~m}$ ( 21 persons).

In constrast the ventilation rate in the chambre in the valley $(540 \mathrm{~m})$ increased by the amount of $17,3 \%$ when the $\mathrm{O}_{2}$-tension was reduced to the pressure of Jungfraujoch.

The "normalisation" of the alveolar $\mathrm{CO}_{2}$ by breathing pure oxygen in an underpressurechambre (Haldane's objection) may not actually support his view, because the much stronger reduction of the ventilation (compared with a normal ventilation-rate in normal air (own observations!) was not measured, allthough this is the real cause of this phenomenon.

Similarly, by artificially normalising the $\mathrm{O}_{2}$ pressure to equal valley-conditions, we found a much larger burning time for candle flames under pressure of $1 / 2$ atmosphere compared with the shorter burning-time in the valley.

These and other experiences support partially Mosso's view.

Prof. Dr. med. Karl Lenggenhager

Erlachstraße 19

CH-3012 Bern 